\title{
Una necesidad no cubierta en Ecuador: programa, estrategia y desafíos de ECMO; experiencia inicial en un centro
}

\author{
An unmet need in Ecuador: ECMO program, strategy and challenges; \\ a single-center experience
}

Uma necessidade não atendida no Equador: programa, estratégia e desafios de ECMO; experiência inicial em um centro

\author{
Mónica Gilbert Orús (iD) a Juan Javier Zúñiga Bohórquez (D) a, Eliana Cerón López (D) a,b, \\ Luciana Boloña Gilbert (iD c, Ivon Coello Silva (D) d, Paola Alejandra Morejón Barragán (iDe
}

\begin{abstract}
a Servicio de Cirugía Cardiovascular y Torácica, Clínica Guayaquil, Guayaquil-Ecuador; b Perfusión Cardiovascular, Clínica Guayaquil, Guayaquil-Ecuador, c Servicio de Cirugía General, Clínica Guayaquil, Guayaquil - Ecuador; d Servicio de Enfermería en Unidad de Cuidados Críticos, Clínica Guayaquil, Guayaquil - Ecuador; e Servicio de Insuficiencia Cardiaca Avanzada, Asistencia Circulatoria y Trasplante Cardiaco, Clínica Guayaquil, Guayaquil-Ecuador.
\end{abstract}

\section{Correspondencia a:}

Mónica Gilbert Orús, mdgilbert@clinicaguayaquil.com

Recibido: 17 de mayo, 2021

Aceptado: 21 de mayo, 2021 Publicado: 1 de junio, 2021

\section{ARTÍCULO ORIGINAL}

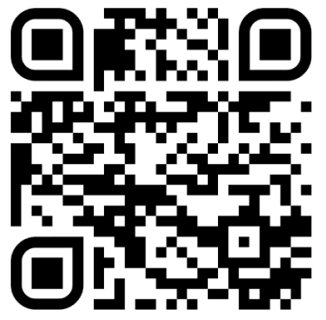

Escanea en tu dispositivo móvil o revisa este artículo en: https:// revistaclinicaguayaquil.org

\section{RESUMEN}

Introducción: El uso de la oxigenación por membrana extracorpórea (ECMO) constituye una alternativa de tratamiento para pacientes seleccionados en casos de síndrome de distrés respiratorio agudo y/o shock cardiogénico. El objetivo del siguiente estudio es describir las características y los resultados de los pacientes asistidos con ECMO en las fases iniciales del programa hospitalario.

Materiales y Métodos: Se presenta una serie de 6 pacientes consecutivos atendidos en el Hospital Clínica Guayaquil, luego de la instauración del programa de ECMO, en abril de 2021.

Resultados: Se incluyeron 4 pacientes con síndrome de distrés respiratorio del adulto y 2 pacientes con shock cardiogénico, reportando una tasa de destete exitoso del $80 \%$ de 5 casos $(n=4)$. No se presentaron complicaciones de sangrado o trombosis, ni necesidad de recambio de membrana o circuito.

Conclusiones: El ECMO representa una herramienta para el tratamiento de la insuficiencia respiratoria severa y/o shock cardiogénico, en pacientes seleccionados.

Palabras clave: ECMO; Oxigenación por Membrana Extracorpórea; COVID-19; choque cardiogénico; reanimación cardiopulmonar

\begin{abstract}
Introduction: The use of extracorporeal membrane oxygenation (ECMO) constitutes an alternative treatment for selected patients in cases of acute respiratory distress syndrome and/or cardiogenic shock. The purpose of the following study is to describe the characteristics and results of patients assisted with ECMO in the initial experience of the program.

Materials and Methods: We present a series of 6 consecutive patients treated at Hospital Clínica Guayaquil, after the establishment of the ECMO program, in April 2021.

Results: Four patients with adult respiratory distress syndrome and 2 patients with cardiogenic shock were included, reporting a successful weaning rate of $80 \%$ of 5 cases $(n=4)$. There were no complications of bleeding or thrombosis, nor the need for membrane or circuit replacement.

Conclusions: ECMO represents a tool for the treatment of severe respiratory failure and/or cardiogenic shock in selected patients.
\end{abstract}

Key words: ECMO; Extracorporeal Membrane Oxygenation; COVID-19; cardiogenic shock; cardiopulmonary resuscitation 


\section{RESUMO}

Introdução: O uso de oxigenação por membrana extracorpórea (ECMO) constitui uma alternativa de tratamento para pacientes selecionados em casos de síndrome do desconforto respiratório agudo e / ou choque cardiogênico. O objetivo do estudo a seguir é descrever as características e os resultados dos pacientes atendidos com ECMO na experiência inicial do programa.

Materiais e Métodos: Apresentamos uma série de 6 pacientes consecutivos atendidos no Hospital Clínica Guayaquil, após o estabelecimento do programa de ECMO, em abril de 2021.

Resultados: Quatro pacientes com síndrome do desconforto respiratório adulto e 2 pacientes com choque cardiogênico foram incluídos, relatando uma taxa de desmame bem-sucedida de $80 \%$ de 5 casos $(n=4)$. Não houve complicações de sangramento ou trombose, nem necessidade de troca de membrana ou circuito.

Conclusões: A ECMO representa uma ferramenta para o tratamento da insuficiência respiratória grave e / ou choque cardiogênico em pacientes selecionados.

Palavras-chave: ECMO; Oxigenação por Membrana Extracorpórea; COVID-19; choque cardiogénico; reanimação cardiopulmonar

\section{INTRODUCCIÓN}

El mundo ha experimentado grandes desafíos a partir de diciembre del 2019, la nueva situación creada por la pandemia generó una serie de recomendaciones, y las terapias avanzadas no se quedaron al margen. Es así que el uso de la oxigenación por membrana extracorpórea (ECMO, por sus siglas en inglés) surgió como una alternativa segura de tratamiento para pacientes seleccionados en el escenario del síndrome de distrés respiratorio agudo (SDRA). A nivel internacional, a través del registro de ELSO (Extracorporeal Life Support Organization), se pueden conocer los resultados de esta terapia. A pesar de la situación local, ningún centro en Ecuador reportaba a la fecha datos y esta terapia parecía no ser una alternativa real en el país.

El presente escrito tiene como objetivo describir las característicasy los resultados de los pacientes asistidos con ECMO en las fases iniciales del programa instaurado en la Institución.

\section{MATERIALES Y MÉTODOS}

Se realizó una revisión retrospectiva de los seis casos admitidos en el servicio de ECMO del Hospital Clínica Guayaquil desde abril de 2021.

\section{RESULTADOS}

\section{Caso 1}

Paciente masculino de 52 años con neumonía por SARS-CoV-2, trasladado desde un hospital de la ciudad de Guayaquil a la Clínica Guayaquil, por presentar índices $\mathrm{PaO}_{2} / \mathrm{FiO}_{2}(\mathrm{PaFi})<100$ asociado a hipercapnia durante 18 horas a pesar de soporte ventilatorio invasivo y 2 ciclos de pronación previos, referido como candidato a soporte con ECMO. Se realizó implante inmediato a su arribo de ECMO VV por presentar PaFi de 47 y $\mathrm{pCO}_{2}$ de $69.9 \mathrm{mmHg}$. Se constató su ingreso, previo al implante del dispositivo, disfunción renal aguda con requerimiento de terapia sustitutiva renal. Por vía aérea dificultosa, se decidió traqueostomía temprana dentro de las primeras 72 horas de soporte. Durante el tiempo de asistencia presentó complicaciones menores relacionadas con sangrado de la traqueostomía y neumonía bacteriana, sin que generen alteraciones hemodinámicas. Se realizó el destete exitoso del soporte con ECMO al día 14 de dicha terapia. El paciente recuperó la función renal 4 días posteriores al retiro del soporte respiratorio. A los 7 días del destete, el paciente falleció debido a tromboembolismo pulmonar masivo.

\section{Caso 2}

Paciente masculino de 65 años trasladado a la Institución con diagnóstico de COVID-19, con 5 días deasistenciarespiratoria mecánica(ARM) por SDRA, con parcial mejoría durante las primeras 24 horas de decúbito prono; sin embargo, por hipoxemia refractaria con PaFi menor de 80 se decide inicio de soporte respiratorio con ECMOV. A las 72 horas se realizó traqueostomía. Durante los 23 días de soporte respiratorio intercurrió con neumonía bacteriana, con buena respuesta al esquema antibiótico instaurado. Posteriormente, presentó delirium hiperactivo y miopatía severa. Constituye el caso de mayor tiempo bajo soporte respiratorio de esta serie. Es dado de alta a los 27 días del destete.

\section{Caso 3}

Primer ECMO móvil realizado en Ecuador desde una ciudad localizada a $71 \mathrm{~km}$ de la ciudad de Guayaquil. Paciente masculino de 35 años con diagnóstico de COVID-19, quien en las 24 horas previas presentóneumomediastino con dificultad para la intubación orotraqueal, motivo por el 
cual se había realizado una cricotiroidotomía de emergencia; posteriormente presentó dificultad para lograr parámetros protectivos de ventilación mecánica, presentando presiones pico de 35 - $40 \mathrm{mmHg}$. Durante el traslado inicial, por parte del hospital base hacia la ciudad de Guayaquil, el paciente presentó inestabilidad hemodinámica y desaturación severa, motivo por el cual no es transportado. Consecuentemente, se decide realizar implante de ECMO VV en la institución de origen y posterior traslado a la Clínica Guayaquil. Intercurre a las 12 horas con neumotórax espontáneo con requerimiento de tubo de avenamiento pleural. El cuadro clínico progresa y se acompaña de vasoplegía refractaria; a los 7 días se realizó diagnóstico de candidiasis sistémica, por lo que se declara futilidad de soporte.

\section{Caso 4}

Paciente masculino de 47 años con neumonía por SARS-COV-2, con requerimiento de ARM hace 48 horas, deterioro de la PaFi e incapacidad de mantener parámetros protectivos en el ventilador. Constituye el segundo traslado realizado con ECMO implantado en otra institución de la ciudad Guayaquil, Ecuador. Por la evolución evidenciada durante las primeras 72 horas, no se considera la realización de traqueostomía. El paciente es asistido con ECMO VV durante 8 días. A las 24 horas de realizado el destete de ECMO, luego de aproximadamente una semana de soporte, se procedió con desvinculación de soporte ventilatorio mecánico; no presentó intercurrencias durante su hospitalización. Se mantiene en seguimiento ambulatorio.

\section{Caso 5}

Paciente masculino de 63 años con diagnóstico de cardiopatía isquémica y disfunción sistólica severa del ventrículo izquierdo, quien en el postoperatorio inmediato de cirugía de revascularización miocárdica intercurre con shock cardiogénico requiriendo implante de balón de contrapulsación intra aórtico (BCIA) a las 8 horas, con parcial mejoría. Sin embargo, a las 24 horas presenta signos de falla del ventrículo derecho y se decide escalar el soporte a ECMO-VA (Figura 1). A las 72 horas se objetiva en el control ecocardiográfico mejoría de la función ventricular, por lo que se optimizan parámetros de soporte. No se presentaron complicaciones vasculares, de sangrado o neurológicas durante el mantenimiento. Se realizó el destete satisfactorio a los 8 días de asistencia con ECMO VA, y 24 horas después, se descontinuó el BCIA. Intercurrió con síndrome hepatorrenal a los 4 días de retiro del soporte circulatorio. Al momento se mantiene en seguimiento por ambulatorio y en titulación de medicación crónica por insuficiencia cardiaca con fracción de eyección reducida.

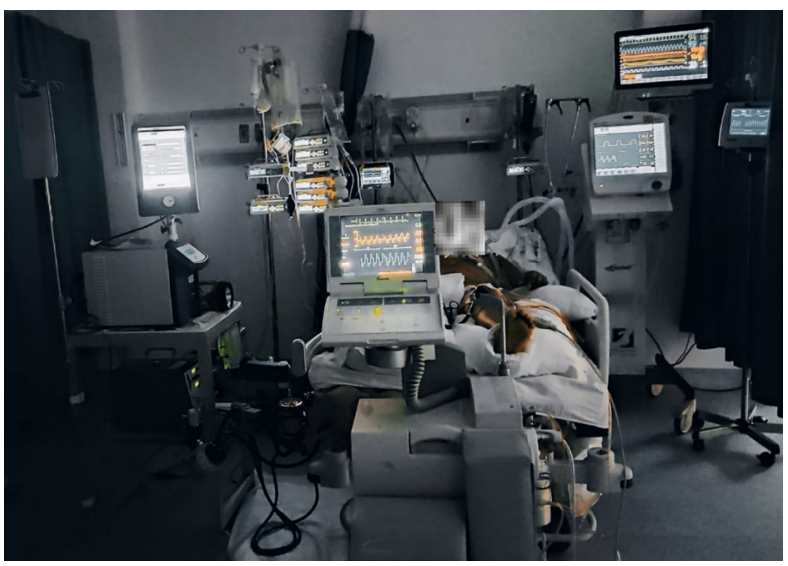

Figura 1. Disposición de equipamiento en paciente asistido con ECMO-VA por shock cardiogénico (Cortesía de Mónica Tovar, MD).

\section{Caso 6}

Paciente masculino de 46 años, quien al momento del presente estudio se encontraba bajo soporte circulatorio. Corresponde al primer caso de soporte en el contexto de paro cardiorrespiratorio. A su ingreso, se diagnosticó con síndrome coronario agudo con elevación del segmento ST de 4 horas de evolución; por patrón electrocardiográfico se sospecha de compromiso del tronco de la arteria coronaria izquierda. Presentó insuficiencia ventilatoria a los 30 minutos del ingreso, con posterior parada cardiorrespiratoria sin recuperación de la circulación espontánea a pesar de las maniobras de resucitación convencional, por lo cual, a los 35 minutos se inicia resucitación cardiopulmonar con soporte con ECMO-VA, también denominado, RCP-extracorpórea (e-RCP). En el estudio por cateterismo cardiaco, se evidenció enfermedad coronaria crónica y oclusión total del tronco de coronaria izquierda; se realizó angioplastia primaria exitosa. Al momento del implante, el objetivo del soporte fue como puente a la recuperación.

En los 6 casos se realizó control de la anticoagulación con tiempo de coagulación activado (ACT, activated clotting time) y tiempo de tromboplastina parcial (TTP), sin presentar complicaciones de sangrado o trombosis. Ningún 
caso requirió cambio de membrana. A la fecha, no se han evidenciado complicaciones relacionadas a los dispositivos de soporte implementados. En la tabla 1 se detallan características de cada uno de los casos.

Tabla 1. Características de la población.

\begin{tabular}{lcccccc}
\hline Edad & 1 & 2 & 3 & 4 & 5 & 6 \\
\hline Género & 52 & 65 & 35 & 47 & 63 & 46 \\
\hline $\begin{array}{l}\text { Enfermedad de } \\
\text { base }\end{array}$ & Masculino & Masculino & Masculino & Masculino & Masculino & Masculino \\
\hline
\end{tabular}

\begin{tabular}{|c|c|c|c|c|c|c|}
\hline $\begin{array}{l}\text { Antecedentes } \\
\text { patológicos } \\
\text { personales }\end{array}$ & HTA & HTA & - & Asma & HTA & Dislipidemia \\
\hline PaFi pre ECMO* & 47 & 74 & 73 & 103 & N/A & N/A \\
\hline $\begin{array}{l}\text { Tiempo de ARM } \\
\text { pre ECMO (días) }\end{array}$ & 2 & 7 & 2 & 1 & $\begin{array}{c}0.75 \\
\text { (18 horas) }\end{array}$ & $\begin{array}{c}0.17 \\
\text { (4 horas) }\end{array}$ \\
\hline Tipo de ECMO & & Veno-venos & & & Veno-arterial & $\begin{array}{c}\text { Veno-arterial } \\
(\mathrm{e}-\mathrm{RCP})\end{array}$ \\
\hline ECMO móvil & No & No & $\mathrm{Si}$ & $\mathrm{Si}$ & No & No \\
\hline $\begin{array}{l}\text { Tipo de } \\
\text { canulación }\end{array}$ & Fémc & oro-Yugular (pe & rcutánea) & & $\begin{array}{l}\text { Fémoro-Femoral } \\
\text { (percutánea) }\end{array}$ & $\begin{array}{l}\text { Fémoro-Femoral } \\
\text { (percutánea) }\end{array}$ \\
\hline Centrífuga & Bio-Console ${ }^{\mathrm{TM}}$ & Cardiohelp & $\begin{array}{l}\text { Bio- } \\
\text { Console }^{\mathrm{TM}}\end{array}$ & $\begin{array}{c}\text { Bio- } \\
\text { Console }^{\mathrm{TM}}\end{array}$ & Bio-Console $^{\mathrm{TM}}$ & Bio-Console ${ }^{\mathrm{TM}}$ \\
\hline $\begin{array}{l}\text { Membrana } \\
\text { oxigenadora }\end{array}$ & $\begin{array}{l}\text { A.L.ONE ECMO } \\
\text { oxygenator adulto } \\
\text { (EUROSETS) }\end{array}$ & $\begin{array}{c}\text { HLS Set } \\
\text { Advanced } \\
5.0 \text { (Maquet) }\end{array}$ & \multicolumn{4}{|c|}{ A.L.ONE ECMO oxygenator adulto (EUROSETS) } \\
\hline RESP score & 3 & 2 & 1 & 5 & SAVE -6 & SAVE -10 \\
\hline $\begin{array}{l}\text { Complicaciones } \\
\text { relacionadas con } \\
\text { el dispositivo }\end{array}$ & \multicolumn{5}{|c|}{ Ninguna } & $\begin{array}{c}\text { En soporte } \\
\text { actualmente }\end{array}$ \\
\hline Días en soporte & 14 & 23 & 7 & 8 & 8 & 2 \\
\hline Destete exitoso & $\mathrm{Si}$ & $\mathrm{Si}$ & Futilidad & $\mathrm{Si}$ & $\mathrm{Si}$ & $\begin{array}{l}\text { En soporte } \\
\text { actualmente }\end{array}$ \\
\hline $\begin{array}{l}\text { Mortalidad } \\
\text { intrahospitalaria } \\
\text { (causa) }\end{array}$ & $\begin{array}{c}\mathrm{Si} \\
\text { (Tromboembolismo } \\
\text { pulmonar masivo } \\
\text { + sangrado de vía } \\
\text { aérea) }\end{array}$ & No & $\begin{array}{l}\text { Si } \\
\text { (Sepsis por } \\
\text { Candida } \\
\text { spp) }\end{array}$ & No & No & $\begin{array}{c}\text { En soporte } \\
\text { actualmente }\end{array}$ \\
\hline $\begin{array}{l}\text { En seguimiento } \\
\text { ambulatorio }\end{array}$ & N/A & $\mathrm{Si}$ & N/A & $\mathrm{Si}$ & $\mathrm{Si}$ & N/A \\
\hline
\end{tabular}

* En casos de ECMO VV por distrés respiratorio.

ARM: asistencia respiratoria mecánica, ECMO: oxigenación por membrana extracorpórea, e-RCP: reanimación cardiopulmonar extracorpórea, HTA: hipertensión arterial, N/A: no aplica, SAVE: Survival after Veno-Arterial ECMO (supervivencia luego del soporte con ECMO Veno-Arterial), SCA: síndrome coronario agudo.

\section{DISCUSIÓN}

Al momento, en la literatura médica no figuran reportes de la experiencia de un programa de ECMO en Ecuador, este estudio se convirtió en el primero que hace referencia al servicio antes mencionado a nivel nacional. El Hospital Clínica Guayaquil se convierte en el primer centro de salud en Ecuador en reportar a la
ELSO, sociedad internacional rectora del área, quien dicta los parámetros para el desarrollo de programas de ECMO. Tal como lo indican en su portal web en donde se encuentran detalladas las diferentes guías (1), en su última versión, especifican que un centro ECMO mínimo debe implantar 6 dispositivos al año y debería 
participar activamente en ELSO, destacando así la característica multidisciplinaria, con diferentes roles y responsabilidades dentro del programa, así como además, la importancia del entrenamiento como pilar en los integrantes del equipo (2) para selección, canulación, manejo y cuidado del paciente en ECMO.

Son más de 40 años del desarrollo de ECMO como terapia, inicialmente enfocada en el paciente crítico con falla respiratoria, por lo que domina la configuración veno-venosa. Esta terapia se ha expandido rápidamente gracias a los avances tecnológicos y a la demanda acelerada por la pandemia que actualmente afecta al mundo (3). En el Hospital Clínica Guayaquil, una vez que se reunieron las sugerencias con respecto del staff médico, se dio inicio al programa.

A la fecha, varias son las publicaciones sobre ECMO en COVID-19 (4-7); el registro de ELSO cuenta con más de 7200 pacientes que requirieron esta terapia, con una mortalidad del 49\%. Al momento, Latinoamérica aporta con 490 casos (8). El requerimiento de soporte cardiaco en esta cohorte es sólo del 4\% (8); acorde con la experiencia inicial de los autores no se ha presentado el escenario clínico para conversión de soporte. En este estudio se encontró que un $80 \%$ de weaning satisfactorio y una mortalidad bajo soporte del $25 \%$, marcada por futilidad. Llamativamente, menos del 30\% del registro internacional de COVID-19 de la ELSO son mujeres (8), lo que concuerda con esta investigación, en la cual no se encuentran casos de pacientes de sexo femenino.

Por otro lado, el soporte con ECMO-VA, indicado ante la presencia de falla cardiaca o insuficiencia respiratoria con compromiso hemodinámico o falla del ventrículo derecho, es otra configuración que brinda soporte circulatorio y respiratorio de manera inmediata. Aunque la evidencia de estudios randomizados es esquiva en esta área, el ECMO-VA ha sido adoptado en los diferentes programas de asistencia circulatoria por su versatilidad y accesibilidad, particularmente en la región latinoamericana. Para el 2018, los datos del registro internacional reportaron un crecimiento del número de casos en soporte con ECMO-VA de más de $1180 \%$ en la última década (9). Diferentes escenarios han emergido como indicaciones de ECMO-VA o, al menos, para ser consideradas dentro de los equipos.

Los dos casos presentados de ECMO-VA son escenarios desafiantes por la presentación clínica que amerita un accionar inmediato, aún más, si se consideraba la emergente aplicación de ECMO como componente de la reanimación cardiopulmonar (10). En la literatura médica, varios aspectos están en debate, incluyendo la ausencia de resultados definitivos, lo cual puede influir en la toma de decisiones para extender su uso.

Una de las características al momento de desarrollar el programa es el transporte de pacientes en ECMO (conocido también como ECMO Móvil), una vez más las guías de ELSO dictan las pautas (11). Este reporte hace referencia a 2 casos trasladados hacia la Institución por el servicio de ECMO móvil. Las guías y los resultados alcanzados sugieren que, aquellos pacientes con distrés respiratorio severo o shock cardiogénico que cumplan criterios para recibir esta terapia y se encuentran con deterioro progresivo de su cuadro clínico en centros no ECMO, deben ser remitidos de forma temprana para consideración de ECMO y así permitir un transporte seguro o tiempo para organizar el rescate con ECMO móvil (12).

En concordancia con lo descrito anteriormente, los desafíos del inicio de este programa son diversos, incluyendo la falta de estandarización en varios aspectos y la incertidumbre de la propia patología. A pesar de esto, ECMO se ha convertido en una herramienta que cubre las necesidades funcionales de los pacientes con insuficiencia respiratoria $\mathrm{y} / \mathrm{o}$ cardiaca, pero que debe ser utilizada de manera responsable por el impacto económico en los sistemas de salud y desde luego por el paciente, se pueden desarrollar estos programas si se cuenta con la infraestructura, el equipo médico y paramédico calificado con entrenamiento en esta terapia, como las claves para lograr buenos resultados.

\section{REFERENCIAS BIBLIOGRÁFICAS}

1. Extracorporeal Life Support Organization. ELSO GUIDELINES [Internet]. 2021. Disponible en: https://www.elso.org/Resources/Guidelines.aspx

2. Extracorporeal Life Support Organization. ELSO guidelines for ECMO Centers, Version 1.8 [Internet]. Ann Arbor; Disponible en: https://www. elso.org/Portals/0/IGD/Archive/FileManager/faf3f6a3c7cusersshyerdocumentselsoguidelinesecmocentersv1.8.pdf

3. Sauer CM, Yuh DD, Bonde P. Extracorporeal membrane oxygenation use has increased by $433 \%$ in adults in the United States from 2006 to 2011. ASAIO J. 2015;61(1):31-6.

4. Jacobs JP, Stammers AH, Louis JS, Hayanga JWA, Firstenberg MS, Mongero LB, et al. Multiinstitutional Analysis of 100 Consecutive Patients with COVID-19 and Severe Pulmonary 
Compromise Treated with Extracorporeal Membrane Oxygenation: Outcomes and Trends over Time. ASAIO J. 2021;496-502.

5. Badulak J, Antonini MV, Stead CM, Shekerdemian $\mathrm{L}$, Raman L, Paden $\mathrm{ML}$, et al. Extracorporeal Membrane Oxygenation for COVID-19: Updated 2021 Guidelines from the Extracorporeal Life Support Organization. ASAIO J. 2021;485-95.

6. Hunziker $P$, Zenklusen $U$. Lean Ad hoc Extracorporeal Membrane Oxygenation Systems for COVID-19. ASAIO J. 2021;12-7.

7. Jacobs JP, Stammers AH, Louis J, Hayanga JWA, Firstenberg MS, Mongero LB, et al. Extracorporeal Membrane Oxygenation in the Treatment of Severe Pulmonary and Cardiac Compromise in Coronavirus Disease 2019: Experience with 32 Patients. ASAIO J. 2020;722-30.

8. Extracorporeal Life Support Organization. ECMO in COVID-19 [Internet]. 2021. Disponible en: https://www.elso.org/COVID19.aspx

9. Guglin M, Zucker MJ, Bazan VM, Bozkurt B, El
Banayosy A, Estep JD, et al. Venoarterial ECMO for Adults: JACC Scientific Expert Panel. J Am Coll Cardiol [Internet]. 2019;73(6):698-716. Disponible en: https://www.sciencedirect.com/science/ article/pii/S073510971839449X

10. Shin TG, ChoiJH, Jo IJ, Sim MS, Song HG, Jeong YK, et al. Extracorporeal cardiopulmonary resuscitation in patients with inhospital cardiac arrest: A comparison with conventional cardiopulmonary resuscitation. Crit Care Med. 2011;39(1):1-7.

11. Dirnberger $D$, Fiser $R$, Harvey $C$, Lunz D, Baccjetta M, Frenckner B, et al. ELSO Guidelines for ECMO Transport. Extracorporeal Life Support Organization. Ann Arbor; 2015.

12. Shekar K, Badulak J, Peek G, Boeken U, Dalton $\mathrm{HJ}$, Arora L, et al. Extracorporeal Life Support Organization Coronavirus Disease 2019 Interim Guidelines: A Consensus Document from an International Group of Interdisciplinary Extracorporeal Membrane Oxygenation Providers. ASAIO J. 2020;707-21.

\section{ACERCA DE LOS AUTORES}

1. Mónica Gilbert Orús. Servicio de Cirugía Cardiovascular y Torácica, Clínica Guayaquil, Guayaquil-Ecuador.

ORCID: 0000-0002-5861-0237

2. Juan Javier Zúñiga Bohórquez. Servicio de Cirugía Cardiovascular y Torácica, Clínica Guayaquil, Guayaquil-Ecuador.

ORCID: 0000-0001-7777-0845

3. Eliana Cerón López. Enfermera perfusionista del Servicio de Cirugía Cardiovascular y Torácica, Clínica Guayaquil, Guayaquil-Ecuador.

ORCID: 0000-0002-4186-0248

4. Luciana Boloña Gilbert. Jefe de Residentes, Servicio de Cirugía General, Clínica Guayaquil, Guayaquil - Ecuador.

ORCID: 0000-0002-0319-9882

5. Ivon Coello Silva. Coordinadora del Servicio de Enfermería en Unidad de Cuidados Críticos Cardiovasculares, Clínica Guayaquil, Guayaquil Ecuador.

ORCID: 0000-0002-5994-401X

6. Paola Alejandra Morejón Barragán. Servicio de Insuficiencia Cardiaca Avanzada, Asistencia Circulatoria y Trasplante Cardiaco, Clínica Guayaquil, Guayaquil-Ecuador.

ORCID: 0000-0003-4354-3139 